\title{
Les fondements conceptuels et méthodologiques d'une approche cognitive de la poésie
}

The Conceptual and Methodological Foundations of a Cognitive Approach to Poetry

Marc Dominicy

\section{OpenEdition}

Journals

Édition électronique

URL : http://journals.openedition.org/pratiques/4786

DOI : $10.4000 /$ pratiques. 4786

ISSN : 2425-2042

Éditeur

Centre de recherche sur les médiations (CREM)

Référence électronique

Marc Dominicy, « Les fondements conceptuels et méthodologiques d'une approche cognitive de la poésie », Pratiques [En ligne], 179-180 | 2018, mis en ligne le 31 décembre 2018, consulté le 20 avril 2019. URL : http://journals.openedition.org/pratiques/4786 ; DOI : 10.4000/pratiques.4786

Ce document a été généré automatiquement le 20 avril 2019.

(c) Tous droits réservés 


\section{Les fondements conceptuels et méthodologiques d'une approche cognitive de la poésie}

The Conceptual and Methodological Foundations of a Cognitive Approach to Poetry

Marc Dominicy

1 Quoique je n'aie, pour ce qui me concerne directement, aucune compétence en matière de didactique de la poésie depuis la maternelle jusqu'à la terminale, je pense pouvoir m'appuyer sur mon expérience d'enseignant universitaire afin de mettre en lumière les obstacles épistémologiques susceptibles de s'opposer à ce qu'une approche cognitive prenne sa place dans un cursus de langue et de littérature dédié, entre autres choses, à la formation des maitres et des professeurs. D'une certaine manière, j'adopterai, dans ce qui va suivre, le point de vue de Sirius, et l'on pourra me reprocher de me perdre dans des préliminaires avant d'en arriver au thème de cette livraison. Mais je demeure convaincu de l'utilité que revêt pareille démarche, ne serait-ce que pour éviter des débats qui surgiront inévitablement à l'une ou l'autre occasion, sans que les tenants et aboutissants de la discussion soient alors clairs aux yeux de tous.

2 Afin de baliser mon propos, j'aborderai tour à tour les obstacles épistémologiques à surmonter, en associant à chacun d'eux une thèse adverse à laquelle je donnerai délibérément une forme catégorique, apte à dissiper d'entrée de jeu les malentendus les plus tenaces. Au risque de paraitre immodeste, je renverrai très souvent à mes propres travaux, avec l'espoir que l'inventaire bibliographique ainsi fourni illustre les liens conceptuels qui s'instaurent, selon moi, entre les différents problèmes abordés.

\section{Nature et culture}

Le premier obstacle épistémologique réside dans le dualisme entre nature et culture. La thèse adverse maintient que la culture est dans la nature; par conséquent, si une 
approche cognitive vise bel et bien à "naturaliser " la culture, elle n'aboutit pas à une vision plus simpliste de la culture, mais à une conception plus riche de la nature ${ }^{1}$.

Je propose d'examiner, à cet égard, cinq situations concrètes, et historiquement attestées, à partir d'un cadre conceptuel aujourd'hui largement partagé par la communauté scientifique, à savoir la théorie néo-darwinienne de l'évolution (Dominicy, 1984) :

1. Avant l'industrialisation de Manchester, la sélection naturelle, due à la présence d'oiseaux prédateurs, favorisait la variante claire de la phalène du bouleau aux dépens de la variante sombre. Avec la pollution industrielle croissante qui a recouvert les bouleaux d'une suie noirâtre, la variante sombre s'est trouvée favorisée aux dépens de la variante claire jusqu'à ce que cette tendance régresse à son tour avec la baisse de la pollution.

2. Avec le développement des thérapies recourant aux antibiotiques, la sélection naturelle a favorisé les bactéries multirésistantes aux dépens des bactéries sensibles aux traitements.

3. Lors du naufrage du Titanic, la sélection naturelle résultant des normes en matière de sauvetage a favorisé les personnes fragiles ou malades aux dépens des individus en bonne santé.

4. En Nouvelle-Angleterre, la sélection naturelle a favorisé les érables aux dépens d'autres arbres parce que les êtres humains, attirés par la couleur que revêt le feuillage des érables à l'automne, ont planté ou protégé de nombreux individus de cette espèce (Dominicy, 2011, p. 39-40).

5. La découverte et la prescription des trithérapies ont modifié les tendances sélectives qui défavorisaient jusque-là les personnes séropositives.

5 Les exemples (1) et (2) ne suscitent généralement aucun débat. Par contre, (3) et (4) laissent perplexes beaucoup de gens. Pourtant, la culture humaine, qui inclut l'activité industrielle et les pratiques médicales, est bel et bien à l'œuvre dans tous les cas. Une première différence semble tenir au fait qu'en (1) et (2), nous avons affaire à des conséquences involontaires du comportement humain. Mais, si l'on s'y arrête un instant, l'exemple (3) prête à discussion de ce point de vue : les hommes d'équipage qui ont fait passer en premier lieu les personnes fragiles ou malades ne cherchaient pas, en réalité, à déclencher un mécanisme de sélection naturelle; ils se bornaient à appliquer une règle morale. Par ailleurs, la démarche qui consisterait à rejeter les situations où le processus sélectif est un résultat volontairement produit conduirait elle-même à d'évidentes impasses, comme le montre l'exemple (5). Quant à l'exemple (4), il provoque une autre gêne, liée au simple fait que l'attitude religieuse ou morale que nous adoptons vis-à-vis de la Nature (je mets la majuscule à dessein) nous pousse à ne pas réduire les propriétés intrinsèques des autres organismes à des fonctions définies par rapport à nos seuls intérêts - et cela même si nous sommes conscients d'avoir favorisé la survie et la propagation de certaines espèces par le jeu de la sélection ${ }^{2}$. En tout état de cause, l'idée, radicalement nouvelle, d'une évolution provoquée, en fin de compte, par le croisement de deux séries causales (celle des mutations et celle des états possibles de l'environnement) invite à ne pas succomber au dualisme entre nature et culture, et donc à adopter une vision unitariste qui inscrit la culture dans la nature.

\section{Le problème de l'intentionnalité}

6 Le deuxième obstacle épistémologique découle de l'idée selon laquelle les attributions d'états mentaux, parmi lesquels figurent les intentions, échouent à éclairer le sens des textes. Selon la thèse adverse, nous tendons inéluctablement à sous-estimer la complexité 
de la vie mentale telle qu'elle s'exerce quotidiennement, ce qui nous conduit à nous heurter, lors de l'analyse des textes, à des difficultés tout à fait imaginaires.

7 La thèse énoncée dans la section précédente implique que la nature renferme, entre autres entités, les états mentaux des êtres humains - leurs croyances, leurs désirs, leurs intentions, leurs émotions. Je reviendrai plus loin sur les croyances, désirs ou émotions, et je me centrerai, pour l'instant, sur les intentions que nous pouvons entretenir ou que nous pouvons prêter à nos congénères. On touche ici à l'une des interrogations les plus irritantes que suscite presque mécaniquement l'étude de la poésie auprès d'un public non prévenu. Combien de fois n'avons-nous pas entendu objecter, à l'une ou l'autre analyse, que l'auteur "n'a pas pu avoir l'intention» de créer telle ou telle combinaison de voyelles, tel ou tel parallélisme syntaxique, etc. ? Les poéticiens, quant à eux, adhèrent parfois à des doctrines pour le moins étranges: au moment de dénoncer ce qu'ils nomment le "sophisme intentionnel », W. K. Wimsatt et M. C. Beardsley écrivent sans broncher que si un auteur apporte des corrections à son œuvre, cela signifie que l'intention que le premier état parait concrétiser n'était pas son intention ${ }^{3}$. Dans l'un et l'autre cas, le malaise ou le paradoxe provient d'une simple confusion quant à la nature du comportement intentionnel, conçu comme le déroulement d'un plan d'action établi par avance et qui ne saurait subir des altérations que par l'effet d'un ratage ou d'un malentendu. Mais considérons les exemples suivants, empruntés à notre vie la plus ordinaire :

1. Regardant par la fenêtre, je vois des passants marcher sur le trottoir et je leur attribue spontanément un comportement intentionnel - à la différence de ce qui aurait lieu si j'apercevais une personne sénile en train de se mouvoir sans but aucun. Pourtant, je ne sais pas pourquoi (avec quelle intention de plus haut niveau) ces passants se déplacent de la sorte.

2. Le chauffeur passera chez vous vers midi. Il a dû modifier deux fois son itinéraire à cause des travaux sur la rocade.

3. Dimanche passé, j'ai déambulé en ville et je suis finalement rentré chez moi avec un T-shirt aux couleurs du PSG.

4. Hier, je suis allé à l'université en auto tout en réfléchissant à un problème de métrique latine. Je me suis aperçu, au moment de me parquer, que je ne m'étais pas fixé un seul instant sur ma conduite ou sur la circulation ambiante.

8 L'exemple (1) montre que le repérage d'un comportement intentionnel ne nécessite pas l'accès à une planification de haut niveau. De même, nous n'éprouvons aucune peine à identifier un message verbal ou écrit comme intentionnellement produit même si nous n'en saisissons pas la finalité. Par conséquent, si le fait d'attribuer une intention ne suffit pas toujours à donner accès au sens, cela ne résulte pas d'une quelconque inadéquation de l'approche intentionnaliste à l'objet poétique, mais des contraintes qui, de manière plus générale, pèsent sur notre « lecture » du comportement humain. Dans la plupart des situations, nous comprenons d'emblée les faits et gestes de nos congénères. Les problèmes se font jour lorsque l'environnement perturbe les signaux qui doivent nous guider dans cette tâche ou lorsque nous nous trouvons confrontés à des agents insincères. Le premier cas de figure est quotidiennement illustré par la circulation automobile, où la mauvaise qualité, voire l'absence, du contact visuel entre les conducteurs favorise les attributions incorrectes d'intentions. Le deuxième cas de figure apparait quand nous nous fondons sur l'éthos préalable d'un locuteur pour refuser toute négociation a posteriori sur l'intention qu'il déclare avoir entretenue (par exemple, si quelqu'un que nous 
considérons comme un raciste notoire a émis une remarque potentiellement dénigrante à l'encontre d'une personne d'origine étrangère). Dans le domaine de l'interprétation poétique, de nombreuses méprises naissent soit d'une maitrise insuffisante de l'environnement historique et culturel, soit d'une confiance ou d'une défiance excessive vis-à-vis des intentions que s'attribue l'auteur (Cioffi, 1964).

L'exemple (2) illustre la plasticité du comportement intentionnel qui, même s'il procède d'une planification préalable fort précise, s'adapte systématiquement aux circonstances ; nous décelons un défaut de rationalité chez les sujets qui maintiennent à tout prix un plan d'action en conflit avec les vicissitudes de l'environnement. Entre la première et la seconde éditions des Fleurs du Mal, les vers 5-8 de "La Musique» ont subi des changements drastiques (Dominicy, 1998, p. 77-84):

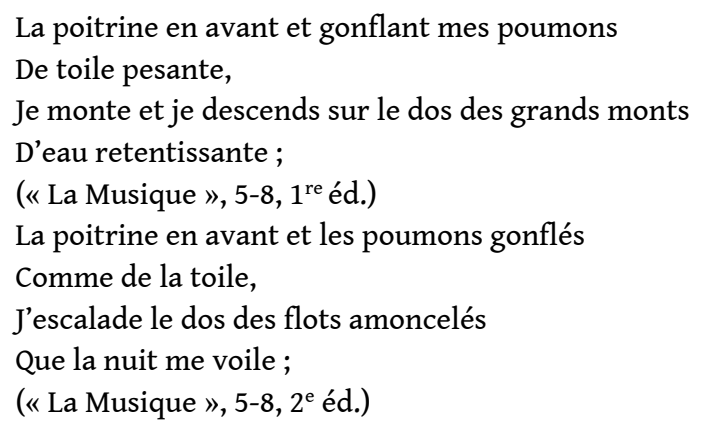

10 Une comparaison avec «Le Beau navire» autorise à penser que, dans l'esprit de Baudelaire, la toile pesante de la version initiale, en tant que trait prototypique, ne saurait entraver le mouvement du navire auquel se trouve assimilé le «Je» envahi par les émotions issues de la musique :

Quand tu vas balayant l'air de ta jupe large,

Tu fais l'effet d'un beau vaisseau qui prend le large,

Chargé de toile, et va roulant

Suivant un rhythme doux, et paresseux, et lent.

( Le Beau navire », 5-8 = 25-28)

Cependant, la toile pourrait être pesante non par nature, mais parce que les vagues l'ont trempée, de sorte qu'il devienne très pénible de naviguer à la voile ; le parallélisme étroit qui unit les vers 6 et 8 ne ferait alors que renforcer cet effet malvenu. Le passage ne soulèverait plus de problème si légère se substituait à pesante, mais la rime l'interdit. Baudelaire a donc modifié son quatrain, et rien ne permet évidemment de soutenir que seule sa réécriture aboutit à réaliser une intention qu'il aurait entretenue dès le départ.

Avec l'exemple (3), nous sommes confrontés à un état de choses où le sujet en question adopte, de manière opportuniste, un comportement que le simple hasard a rendu possible et alors souhaitable. Baudelaire, de nouveau, et Paul Valéry bien malgré lui, nous fournissent une illustration poétique de ce phénomène. Dans la seconde édition des Fleurs du Mal, la pièce c s'ouvre sur la phrase suivante (Dominicy, 2018) :

La servante au grand cœur dont vous étiez jalouse,

Et qui dort son sommeil sous une humble pelouse, Nous devrions pourtant lui porter quelques fleurs.

Pour Valéry (1957-60, II, p. 556),

Ce vers célèbre qui tient tout un roman de Balzac dans ses douze syllabes, - on a été jusqu'à l'expliquer par une histoire de domestique !

La vérité est plus simple. Elle est évidente à un poète - c'est que ce vers est venu à Baudelaire, et il est né avec son air de romance sentimentale - de reproche bête et 
touchant.

Et Baudelaire a continué. Il a enterré la cuisinière dans une pelouse, ce qui est contre la coutume, mais selon la rime, etc. de vers «calculés » où les exigences formelles aboutiraient à ce que la vraisemblance sémantique se trouve mise à mal :

Les dieux, gracieusement, nous donnent pour rien tel premier vers; mais c'est à nous de façonner le second, qui doit consonner avec l'autre, et ne pas être indigne de son aîné surnaturel. Ce n'est pas trop de toutes les ressources de l'expérience et de l'esprit pour le rendre comparable au vers qui fut un don.

(ibid., I, p. 482)

Deux sortes de vers : les vers donnés et les vers calculés.

Les vers calculés sont ceux qui se présentent nécessairement sous forme de problèmes à résoudre - et qui ont pour conditions initiales d'abord les vers donnés, et ensuite la rime, la syntaxe, le sens déjà engagés par ces données.

(ibid., II, p. 551)

Il y a des vers qu'on trouve. Les autres, on les fait.

On perfectionne ceux qu'on a trouvés.

On « naturalise » les autres.

(ibid., II, p. 591) Dans les épreuves de la première édition, les vers 1 et 2 , qui n'étaient séparés par aucune ponctuation, constituaient une structure interrogative du type [syntagme nominal + syntagme verbal], avec enclise sur le verbe (La servante au grand coeur dont vous étiez jalouse / Dort-elle, etc.). Baudelaire a alors ajouté un tiret sur épreuve, afin de transformer le vers 1 en un syntagme nominal détaché à gauche et repris anaphoriquement par l'enclitique elle du vers 2. La première édition porte, peut-être erronément, un second tiret au bout du vers 2 qui fonctionne dès lors comme une incise placée entre le syntagme nominal détaché et sa reprise anaphorique par le proclitique lui du vers suivant. Dans la seconde édition, le vers 2 devient une relative coordonnée à celle qui occupe le second hémistiche $\mathrm{du}$ vers 1 , de sorte que les deux premiers vers constituent maintenant un syntagme nominal détaché à gauche et toujours repris anaphoriquement par le proclitique du vers 3. En bref, l'état initial du texte n'exhibait pas de détachement à gauche, et Baudelaire n'a découvert que peu à peu - voire même de façon opportuniste, par l'entremise d'une coquille - l'effet oratoire qui est déclenché par cette structure syntaxique et qui a sans nul doute créé, chez Valéry, l'illusion d'un « vers donné ».

L'exemple (4) nous rappelle qu'un comportement intentionnel parfaitement mené peut se soustraire à la conscience. Au troisième vers de «La servante au grand cœur... », on lisait d'abord Nous aurions déjà dû lui porter quelques fleurs. Les modifications apportées dans la version définitive créent, outre des effets pragmatiques inédits que j'ai commentés ailleurs, deux particularités formelles. En effet le vers 3 est désormais le seul, de tout le poème, où le mot à l'hémistiche ne relève pas de la catégorie nominale ou de la catégorie verbale, et le fait que pourtant et porter exhibent le même squelette consonantique unit ces deux termes par une motivation relative : le groupe infinitival dont porter est la tête décrit une action que légitime la connexion argumentative exprimée à l'aide de pourtant. Rien n'exige que l'intentionnalité qui a produit ces deux traits ait été accessible à la conscience de Baudelaire. 


\section{Théorie et méthode}

17 Nous nous heurtons au troisième obstacle épistémologique si nous exigeons d'une théorie (cognitive, en l'occurrence) qu'elle fonde une "méthode» sui generis apte à fournir une interprétation empiriquement légitime. La thèse adverse affirme qu'une théorie poétique ne traite que du "principe», jamais du «détail», de sorte que la gamme des interprétations qu'elle autorise pour un texte donné est ouverte, et que seule la philologie permet éventuellement d'atteindre le «détail »4. Amalgamer le niveau du «principe » avec celui du « détail » revient à identifier l'objet d'une production textuelle à la cause dont il procède ; or l'objet ne se confond pas toujours avec la cause. Par ailleurs, une approche cognitive ne saurait être adoptée indépendamment d'une étude préalable des textes envisagés.

18 L'épistémologie nous apprend que les « lois naturelles » ne possèdent aucune capacité prédictive en l'absence de "conditions initiales» qui incluent des clauses en ceteris paribus (Dominicy, 1983). Il en va forcément de même pour les mécanismes de traitement que postule une théorie cognitive de la poésie. Cette limite insurpassable contrarie notre propension invincible à interpréter, et à défendre bec et ongles notre compréhension des textes contre des lectures alternatives que rien n'exclut pourtant dans le "principe ». Lorsqu'il affirme, à propos de la pièce $\mathrm{c}$ des Fleurs du Mal, que le mot pelouse n'est là que pour la rime, Valéry croit ou prétend s'appuyer sur son intuition de locuteur français, qui lui suggèrerait qu'on n'inhume pas sous une pelouse; il y voit une confirmation de la manière dont il a immédiatement perçu l'incipit tel que le donne à lire la version définitive. Mais la philologie démontre qu'il était banal, au XIX ${ }^{\mathrm{e}}$ siècle et au début du XX siècle, de recouvrir les fosses communes de pelouses ou de jardinets ${ }^{5}$; la connaissance que Baudelaire avait, comme tous ses contemporains, de cette réalité fournit ici la cause et l'objet de la proposition relative figurant au vers 2 . Valéry devait bien évidemment partager ce savoir commun; je parierais volontiers qu'ici, sa doctrine poétique a pris le pas sur son expérience du réel. Par ailleurs, sa boutade sur ce qu'il nomme « une histoire de domestique » ne se justifie pas. En effet, l'histoire littéraire nous apprend qu'il faut reconnaitre, dans la servante au grand cœur, une domestique du nom de «Mariette» qui, jeune alors, aurait suscité les premiers émois de Baudelaire; et certaines particularités des pièces XCIX ("Je n'ai pas oublié... ») et c s'expliquent très vraisemblablement par le décès de François Baudelaire pendant l'enfance du poète (1827) et par le triste sort que Madame Aupick réserva à la sépulture de son défunt mari (Dominicy, 2002). De surcroit, la correspondance de Baudelaire avec Madame Aupick durant la période où la mère de Jeanne Duval, décédée et enterrée, sans doute dans la fosse commune, en novembre 1853, fut ensuite exhumée et réinhumée dans une concession de cinq ans durant le mois de décembre qui suivit, jette un éclairage à la fois complexe et cruel sur le contenu moral et émotif du poème. Baudelaire écrit successivement :

C'était hier le dernier délai accordé pour l'accomplissement d'un acte que je regarde comme un Devoir forcé, c'est-à-dire l'exhumation et la réinhumation d'une femme qui m'a donné ses dernières ressources, sans murmurer, sans soupirer, et surtout sans conseiller (26 décembre 1853 ; Baudelaire, 1973, p. 241). Puis :

Tu m'as écrit une lettre bien triste et bien charmante, mais toujours empreinte de ton inguérissable exagération. La femme morte, je l'ai presque haïe. Mais je l'ai laissée mourir dans la plus vraie misère. Et est-ce moi qui ai inventé les préjugés et 
le respect des morts? Ce n'est donc qu'une pure question de convenance (31 décembre 1853 ; ibid., p. 246). a l'expérience ou à la simulation, qui permettent l'une et lautre de maitriser l'impact des «conditions initiales ». Si le défi majeur que doivent relever les approches expérimentales tient à la nécessité qu'il y a de travailler avec des sujets "neutres ", si tant est qu'il en existe, certaines techniques détectent des effets à la fois reproductibles et soustraits à un accès conscient ${ }^{6}$. Là où cette voie ne peut être suivie, il convient d'explorer les structures et mécanismes à l'œuvre à partir d'une description rigoureuse des objets envisagés. Cette exigence réhabilite les "microscopies » qui explorent tous les aspects d'un poème, depuis les traits superficiels auxquels s'attachaient les analyses de Roman Jakobson ou de Nicolas Ruwet jusqu'à la dimension proprement cognitive des actes de langage ${ }^{7}$. Dans ces vers devenus célèbres de W. B. Yeats ${ }^{8}$, la phrase interrogative située en clôture se laisse interpréter soit (dans sa lecture « directe » ou « littérale ») comme une question, soit (dans sa lecture « indirecte » ou « rhétorique ») comme une affirmation :

o chestnut-tree, great-rooted blossomer,

Are you the leaf, the blossom or the bole?

o body swayed to music, o brightening glance,

How can we know the dancer from the dance?

(«Among School Children »)

Selon la théorie des actes de langage, la question est un "acte illocutoire directif » par lequel le locuteur fournit à son allocutaire une raison de lui dire comment distinguer la danseuse de la danse, tandis que l'affirmation est un « acte illocutoire assertif » par lequel le locuteur fournit à son allocutaire une raison de croire qu'on ne saurait distinguer la danseuse de la danse. En accomplissant l'acte directif, le locuteur accomplit également un «acte locutoire» par lequel il exprime son désir que l'allocutaire lui dise comment distinguer la danseuse de la danse ; en accomplissant l'acte assertif, le locuteur accomplit également un "acte locutoire " par lequel il exprime sa croyance qu'on ne saurait distinguer la danseuse de la danse. L'acte directif et l'acte assertif sont incompatibles : on ne peut fournir à quelqu'un une raison valable de dire comment distinguer la danseuse de la danse si on lui fournit par ailleurs une raison de croire qu'on ne saurait distinguer la danseuse de la danse. Mais rien n'interdit à un locuteur, qui entretient la croyance qu'un certain contenu propositionnel $p$ (ici : «L'allocutaire dit au locuteur comment distinguer

Pratiques, $179-180 \mid 2018$ 
la danseuse de la danse ») est " physiquement impossible » (c'est-à-dire exclu par l'état de la nature), d'exprimer le désir que $p$ soit vrai et donc d'accomplir un acte locutoire tel que l'acte illocutoire correspondant fournirait à l'allocutaire une raison de rendre $p$ vrai. Rien, non plus, n'interdit à un locuteur d'accomplir un acte illocutoire qui fournit à l'allocutaire une raison de croire vrai un certain contenu propositionnel $q$ (ici : « On ne saurait distinguer la danseuse de la danse»), et donc d'exprimer locutoirement la croyance que $q$ est vrai, tout en entretenant le désir que $q$ soit faux. La première attitude relève d'une sorte de volontarisme feint et désespéré ; la seconde, plus intellectualiste, traduit la nostalgie d'un univers conceptuel qui nous reste fermé. Du fait que la vérité de $q$ («On ne saurait distinguer la danseuse de la danse ») entraine la fausseté de $p$ ("L'allocutaire dit au locuteur comment distinguer la danseuse de la danse »), l'acte illocutoire indirect fournirait une raison en faveur de la croyance qui coexiste avec l'accomplissement de l'acte locutoire direct; du fait que la satisfaction de l'acte illocutoire direct entrainerait la vérité de $p$ ( «L'allocutaire dit au locuteur comment distinguer la danseuse de la danse »), et donc la fausseté de $q$ ( On ne saurait distinguer la danseuse de la danse »), le désir locutoirement exprimé lors de l'accomplissement de cet acte illocutoire direct implique le désir qui coexiste avec l'acte illocutoire indirect. Un tel entrelacement ne supprime pas l'incompatibilité illocutoire entre les interprétations directive et assertive, mais il montre que le choix de l'une ou l'autre, si on le cantonne au niveau locutoire, n'exclut en aucune façon l'état mental exprimé dans la lecture concurrente (pour d'autres exemples, empruntés à Antonio Machado et à Pablo Neruda, voir Dominicy, 2004). Il s'ensuit que les prises en compte successives de chaque lecture évoquent, dans l'esprit d'un récepteur qui ne doit même pas s'instituer en allocutaire, des contenus de croyances stéréotypés portant sur les états mentaux que lui ou ses congénères peuvent entretenir face à une certaine classe de situations. En ce sens, le poème vise à susciter une forme d'empathie auprès de ce qu'on pourrait appeler «la communauté des esprits humains ».

\section{Les sciences cognitives comme champ de débats}

24 Le quatrième obstacle épistémologique provient de la croyance (ou du souhait) que les sciences cognitives nous pourvoient de conceptions unanimement acceptées par les spécialistes du domaine. En réalité - c'est la thèse adverse - tout poéticien qui s'inspire des sciences cognitives doit être conscient des discussions et des polémiques qui agitent ce champ disciplinaire, non pour y prendre part (et courir ainsi le risque de s'égarer), mais afin d'en saisir les principaux enjeux.

Les démarches interdisciplinaires ne sont pas toujours dénuées, au départ, d'un optimisme excessif. Ainsi, travaillant sur les rapports de perception visuelle (Dominicy, 2007b), j'ai essayé de mettre en lumière non seulement la communauté d'interrogations qui unit, en l'occurrence, la philosophie de l'esprit et les sciences du langage, mais aussi les deux attentes auxquelles il faut renoncer d'emblée, sous peine de se heurter à de graves frustrations : du côté des sciences du langage, l'espoir de trouver toute faite une théorie de la perception visuelle qui soit généralement acceptée et qui viendrait fonder telle ou telle sémantique des énoncés considérés; du côté des sciences (et surtout de la philosophie) de l'esprit, la tentation d'invoquer certaines particularités des formes et des usages linguistiques pour justifier une analyse conceptuelle qui s'appliquerait à la vision, ou à la conscience que nous pouvons en avoir. 
Pour qui s'intéresse à la poétique dans une perspective cognitive, certains débats revêtent une importance cruciale. Il en va ainsi, par exemple, des argumentations développées depuis Descartes sur l'orientation «top down ", ou «bottom up », qu'il faut assigner aux processus générateurs des émotions : la première branche de cette alternative accorde un primat à l'évaluation axiologique de l'objet considéré, laquelle entraine des modifications physiologiques dans un second temps; la seconde branche inverse ce rapport. D'autres approches, comme celle d'Antonio Damasio (1995), proposent un modèle plus complexe dont je crois, pour ma part, qu'il offre un soubassement robuste à l'étude de la poésie (Dominicy, 2011, p.46-50, p.124-125; 2014). Comme les états (propio)perceptuels (éprouver une brûlure à l'estomac, entendre Pierre, voir Pierre courir...), les « émotions primaires" (être écœuré par une odeur, dégoûté par des rognons...) possèdent un contenu non propositionnel ; elles ne peuvent être entretenues sans se voir attribuer un objet, qui peut d'ailleurs ne pas coïncider avec leur cause. Les " émotions secondaires " (être fier de son canari, ému par Marie..., être fier que la Joconde appartienne à un musée français, être choqué de ce que personne n'ait réagi à tels ou tels propos...) possèdent un contenu qui peut (mais ne doit pas) revêtir une nature propositionnelle; on ne saurait donc les entretenir sans qu'elles se voient attribuer un objet qui, de nouveau, peut ne pas coïncider avec leur cause. Toute émotion secondaire, si elle n'est pas feinte, s'accompagne d'une émotion primaire, elle-même associée à certains états somatiques, à l'instar des états (propio)perceptuels. Il en résulte qu'une approche de la poésie sensible à sa dimension « émotive » n'exclut en rien la prise en compte d'états mentaux complexes, et pourvus d'un contenu conceptuel éventuellement articulé sous la forme d'une proposition.

Plus directement encore, je suis enclin à penser - au risque, encore une fois, de me montrer immodeste - qu'il convient de toujours garder à l'esprit deux visions concurrentes de l'interprétation symbolique. Selon la vision que j'appellerai ici « discontinuiste », qui inspire les travaux déjà anciens de Dan Sperber (1974, 1975, 1979a, 1979b, 1982) et mes propositions en la matière (Dominicy, 2007a, 2009a, 2011), les mécanismes de l'interprétation symbolique n'opèrent pas au même niveau que le traitement on line du langage". Selon la vision " continuiste ", que Dan Sperber et Deirdre Wilson ont fini par privilégier à l'intérieur de la Théorie de la Pertinence (Sperber, 1996 ; Sperber \& Wilson, 1989, 2008 ; Pilkington, 1991, 1992, 2000), l'essence des effets poétiques se situe dans les écarts, ou les désordres, qui viennent modifier, ou perturber, les processus en charge de dériver les implicitations; en d'autres termes, les processus interprétatifs postulés se déroulent au même niveau que le traitement on line. Le poème «Annie » de Guillaume Apollinaire peut nous aider à capter les tenants et aboutissants de cette confrontation théorique (Dominicy, 2011, p. 188-191) :

Sur la côte du Texas

Entre Mobile et Galveston il y a

Un grand jardin tout plein de roses

Il contient aussi une villa

Qui est une grande rose

Une femme se promène souvent

Dans le jardin toute seule

Et quand je passe sur la route bordée de tilleuls

Nous nous regardons

Comme cette femme est mennonite

Ses rosiers et ses vêtements n'ont pas de boutons 
Il en manque deux à mon veston

La dame et moi suivons presque le même rite

Si l'on adopte le traitement standard que la Théorie de la Pertinence réserve à la métaphore, l'énoncé Il contient aussi une villa / Qui est une grande rose véhicule l'implicitation « La villa est, comme les roses, un objet contenu dans le jardin »; on peut y ajouter l'implicitation corollaire que les roses prototypiques sont des exemplaires prototypiques de la catégorie des objets contenus dans le jardin. Il n'y a là aucun écart, ni aucun désordre, qui vienne perturber le traitement on line, mais une bizarre platitude. Par ailleurs, on s'attendrait à ce que la suite du texte parle ensuite de la villa, mais certainement pas des roses ou des rosiers. Si l'on postule que le traitement symbolique n'opère pas au même niveau, les choses s'éclaircissent. Dans la deuxième strophe, le jardin contient la femme, de sorte que celle-ci devient, conformément à un topos éculé, " une rose parmi les roses ». Le second quatrain, quant à lui, inverse cette relation de contenant à contenu. On peut comprendre, à partir de l'organisation linguistique, que la femme possède ses rosiers comme elle possède ses vêtements. Mais si l'absence de boutons et de boutonnières n'étonne pas (les mennonites voyant en eux un souvenir de l'uniforme guerrier), on s'explique mal que, dans un tel jardin, les rosiers ne portent pas de boutons; par ailleurs, l'allusion à un « rite » donne au présent n'ont pas une valeur générique qui exclut la lecture où, suite à leur épanouissement, les rosiers auraient perdu (« n'auraient plus») leurs boutons. L'énigme ainsi créée oriente l'interprète vers un sens voilé, de nature sexuelle.

\section{BIBLIOGRAPHIE}

BAUdelaire, C. (1973). Correspondance. Paris : Gallimard.

CIOFFI, F. (1964). «Intention and Interpretation in Criticism ». Proceedings of the Aristotelian Society 64, p. 85-106.

DAMASIO, A. R. (1995) [1994]. L'erreur de Descartes. La raison des émotions. Trad. de l'anglais (ÉtatsUnis) par M. Blanc. Paris : Odile Jacob.

DOMINICY, M. (1983). «Falsification and Falsifiabilization: From Lakatos to Goodman ». Revue internationale de philosophie 144-145, p. 163-197.

Dominicy, M. (1984). « Darwin, Saussure et les limites de l'explication ». In : Auroux, S. et al. (éds), Matériaux pour une histoire des théories linguistiques. Lille : Presses universitaires de Lille, p. 553-561. Dominicy, M. (1990). « Prolégomènes à une théorie générale de l'évocation ». In : Vanhelleputte, M. (éd.), Sémantique textuelle et évocation. Louvain : Peeters, p. 9-37.

Dominicy, M. (1998). « Pour une étude linguistique des variantes : l'exemple des Fleurs du Mal ». In : Hausmann, F.-R. et al. (éds), Haben sich Sprach- und Literaturwissenschaft noch etwas zu sagen? Bonn : Romanistischer Verlag, p. 69-93.

Dominicy, M. (2002). « De Baudelaire à Montale : étude d'une démarcation » In : Bartning, I. et al. (éds), Mélanges publiés en hommage à Gunnel Engwall, Stockholm, Almqvist \& Wiksell, p. 127-136. 
Dominicy, M. (2003). « Le raisonnement pratique met-il en œuvre une nécessité logique ? Une critique de Georg Henrik von Wright ». In : Zaccaï-Reyners, N. (éd.), Explication - compréhension. Regards sur les sources et l'actualité d'une controverse épistémologique. Bruxelles : Éd. de l'Université de Bruxelles, p. 185-202.

Dominicy, M. (2004). « La pregunta poética ». Cuadernos Hispanoamericanos 643, p. 15-22. En ligne : http://www.cervantesvirtual.com/obra/cuadernos-hispanoamericanos--18/.

Dominicy, M. (2007a). «L'évocation discursive. Fondements et procédés d'une stratégie “opportuniste” ». Semen 24, p. 145-165. En ligne : https://journals.openedition.org/semen/6513.

Dominicy, M. (2007b). «Sémantique et philosophie de l'esprit : les rapports de perception visuelle ». In : Neveu, F. \& Pétillon, S. (éds), Sciences du langage et sciences de l'homme. Actes du colloque 2005 de l'Association des Sciences du langage. Limoges : Lambert-Lucas, p. 65-82.

Dominicy, M. (2009a). « Discourse Evocation: Its Cognitive Foundations and Its Role in Speech and Texts ». In : De Brabanter, P. \& Kissine, M. (éds), Utterance Interpretation and Cognitive Models. Bingley : Emerald Group Publishing, p. 179-210.

Dominicy, M. (2009b). « La théorie des actes de langage et la poésie ». L'Information grammaticale 121, p. 40-45. En ligne : https://www.persee.fr/doc/igram_0222-9838_2009_num_121_1_4022.

Dominicy, M. (2011). Poétique de l'évocation. Paris : Classiques Garnier.

Dominicy, M. (2014). «Émile Benveniste et le paradigme stylistique ». Poétique 175, p. 135-156.

Dominicy, M. (2018). « La servante au grand cœur... (poème C des Fleurs du Mal) ». Revue romane en ligne. DOI: https://doi.org/10.1075/rro.16004.dom.

Dominicy, M. (2019). « L'énonciation lyrique et la théorie des actes de langage ». In : Biglari, A. \& Watteyne, N. (éds), Scènes d'énonciation de la poésie lyrique moderne. Paris : Classiques Garnier, p. 41-59

PILKInGton, A. (1991). « Poetic Effects: A Relevance Theory Perspective ». In : Sell, R. D. (éd.), Literary Pragmatics. Londres/New York : Routledge, p. 44-61.

PILKINGTON, A. (1992). « Poetic Effects ». Lingua 87, p. 29-51.

PILKIngton, A. (2000). Poetic Effects: A Relevance Theory Perspective. Amsterdam: John Benjamins. RUWET, N. (1975). « Parallélismes et déviations en poésie ». In : Kristeva, J. et al. (éds), Langue, discours, société. Pour Émile Benveniste. Paris : Éd. du Seuil, p. 307-351.

SPERBER, D. (1974). Le symbolisme en général. Paris : Hermann.

SPERBER, D. (1975). « Pourquoi les animaux parfaits, les hybrides et les monstres sont-ils bons à penser symboliquement?». L'Homme 15, p. 5-34. En ligne : https://www.persee.fr/doc/ hom_0439-4216_1975_num_15_2_367550.

SPERBER, D. (1979a). « La pensée symbolique est-elle pré-rationnelle? ». In : Izard, M. \& Smith, P. (éds), La fonction symbolique. Essais d'anthropologie. Paris : Gallimard, p. 17-42.

SPERBER, D. (1979b). « Remarques sur l'absence de contribution positive des anthropologues au problème de l'innéité ». In : Piattelli-Palmarini, M. (éd.), Théories du langage. Théories de l’apprentissage. Paris : Éd. du Seuil, p. 361-365.

SPERBER, D. (1982). Le savoir des anthropologues. Paris : Hermann.

SPERBER, D. (1996). La contagion des idées. Théorie naturaliste de la culture. Paris : Odile Jacob. 
SPERBER, D. \& WILSON, D. (1989). La pertinence. Communication et cognition. Trad. de l'anglais par A. Gerschenfeld \& D. Sperber. Paris : Éd. de Minuit.

SPERBER, D. \& WILSON, D. (2008). « A Deflationary Account of Metaphor ». In : Gibbs R. W. Jr. (éd.), The Cambridge Handbook of Metaphor and Thought. Cambridge: Cambridge University Press, p. 84-105.

SWINNEY, D. A., (1979). « Lexical Access during Sentence Comprehension : (Re)Consideration of Context Effects ». Journal of Verbal Learning and Verbal Behavior 18, p. 645-659.

VALÉRY, P. (1957-60). Cuvres. Paris : Bibliothèque de la Pléiade.

WIMSATT, W. K. Jr. (1970) [1954]. The Verbal Icon: Studies in the Meaning of Poetry. Londres : Methuen.

\section{NOTES}

1. Cette prise de position a pour conséquence que je rejette le dualisme entre « explication » et « compréhension » (Dominicy, 2003).

2. Darwin lui-même distinguait la sélection « naturelle » de la sélection « artificielle » pratiquée par les éleveurs. Mais il s'agissait, chez lui, d'une manœuvre argumentative qui poursuivait deux objectifs très différents. D'un côté (c'est là le versant positif de l'analogie), les résultats de l'élevage confortaient son hypothèse, par ailleurs correcte, selon laquelle un mécanisme sélectif s'appliquant à des individus peut déboucher sur une évolution de l'espèce ; et cela se révélait d'autant plus crucial pour lui qu'il ne disposait d'aucune théorie adéquate de l'hérédité (il n'a jamais eu connaissance des travaux de Mendel). D'un autre côté (versant négatif de l'analogie), il lui fallait mettre en exergue l'absence, dans les processus "naturellement " sélectifs, de la téléologie qui préside aux pratiques des éleveurs.

3. " He intended to write a better work, or a better work of a certain kind, and now has done it. But it follows that his former concrete intention was not his intention " (Wimsatt \& Beardsley [1946] dans Wimsatt 1970: 5). Sur le «sophisme intentionnel» en général, voir l'analyse pénétrante de F. Cioffi (1964) ainsi que M. Dominicy (2011, p. 28, 72-75).

4. Sur la différence entre le « principe » et le « détail », voir N. Ruwet (1975), M. Dominicy (1990, p. 10-13).

5. Voir les passages de Sully Prudhomme et d'Émile Zola cités dans M. Dominicy (2018). Benoît de Cornulier me signale encore ce texte de Paul Arène : "Avez-vous vu la fosse commune ? C'est la partie du cimetière la plus pittoresque, la plus attendrissante: des fleurs, de la verdure; et pressées dans l'herbe, une foule de petites grilles de bois peint en noir entourent chacune son jardinet ", Les Annales politiques et littéraires 906, 4 nov. 1900, p. 299 (en ligne: https:// gallica.bnf.fr/ark:/12148/bpt6k58525902/f11.item).

6. Un exemple classique, et pertinent pour la poétique (voir note 9), nous est fourni par le paradigme expérimental de D. A. Swinney (1979). En gros, les sujets écoutent de courtes histoires dans lesquelles apparait un mot possédant deux sens distincts - en l'occurrence, le substantif anglais bug mis au pluriel, qui peut désigner, entre autres choses, des micros utilisés comme dispositif d'écoute ou des insectes (français «punaises»). Il est chaque fois question d'une chambre où se trouvent des bugs, mais selon le groupe testé, l'histoire porte sur un épisode d'espionnage ou sur la présence malvenue de punaises. Lorsque l'histoire est presque terminée, trois séquences de lettres apparaissent à l'écran et les sujets doivent décider, pour chacune, s'il s'agit ou non d'un mot. La première séquence est adaptée au contexte de l'histoire écoutée ; la deuxième est inadaptée à ce même contexte; la troisième n'entretient aucune relation que ce soit avec lui. Par exemple, si l'histoire concerne un épisode d'espionnage, on aura spy (« espion »), ant (« fourmi ») et sew (« coudre »). On s'attendrait à ce que la reconnaissance de mot 
soit facilitée, dans ce cas, pour spy, mais non pour ant et sew; or la facilitation opère également pour ant, du moins durant un certain laps de temps au-delà duquel la différence entre ant et sew ne subsiste plus. En d'autres termes, à une première étape du traitement, les deux sens de bugs sont activés indépendamment du contexte, et cela sans que le sujet s'en montre conscient ; après coup, un mécanisme de véto élimine la signification inopportune.

7. Sur l'application de la théorie des actes de langage à la poésie, voir M. Dominicy $(2004,2009 \mathrm{~b}$, 2011, 2019).

8. "Ô châtaignier fleuri aux racines profondes, / Qu'es-tu, dis-moi, la feuille ou la fleur ou le tronc? / Ô corps que la musique entraîne, yeux rayonnants, / Comment distinguer la danseuse de la danse?».

9. On peut ainsi penser que le mécanisme de véto qui élimine les significations contextuellement inopportunes (voir note 6) n'opère pas lors du traitement symbolique. À propos de morts abandonnés, Théophile Gautier parle du vague tombeau de ces dormeurs obscurs («La Fontaine du cimetière ", España); le mot vague évoque, simultanément, le vide (latin uacuus, français vaquer), le voyage (latin uagus, français vagabond) et la mer (substantif féminin vague, d'origine germanique) et cette indécision interprétative renvoie à un intertexte hugolien ("Oceano nox ", Les Rayons et les Ombres), où les marins disparus dorm[ent] dans les goëmons verts (Dominicy, 2011, p. 24-25).

\section{RÉSUMÉS}

Cet article traite de quatre obstacles épistémologiques qui peuvent inciter à ne pas introduire les approches cognitives dans un cursus universitaire dédié à l'étude et à l'analyse de la poésie. À chaque fois, l'obstacle en cause se laisse surmonter grâce à des argumentations bien fondées. Le paradigme darwinien appuie l'hypothèse que, loin de soumettre la culture à des réductions simplistes, le cognitivisme enrichit notre conception de la nature. La théorie de l'esprit met en lumière le fonctionnement flexible des états mentaux et des actes de langage, et aide à dissiper les objections émises contre l'intentionnalisme. Cependant, les «principes » d'une approche cognitive ne peuvent produire une interprétation du « détail » textuel sans l'aide de la philologie et de la linguistique. Enfin, les sciences cognitives connaissent de fréquents débats sur des questions pertinentes pour une meilleure compréhension de la poésie.

This paper deals with four epistemological obstacles that may prevent people from introducing cognitive approaches in a university curriculum devoted to the study and analysis of poetry. In each case, the obstacle at hand can be overcome by relying on well-grounded arguments. The Darwinist paradigm supports the claim that, far from submitting culture to simplistic reductions, cognitivism enriches our conception of nature. The theory of mind sheds light on the flexible functioning of mental states and speech acts, and allows dismissing the most frequent objections addressed to intentionalism. Yet the "principles" of a cognitive approach cannot produce any interpretation of the textual "detail" without being coupled with philological and linguistic inquiry. Finally, one should be aware that cognitive scientists frequently disagree on questions that will prove relevant for a better understanding of poetry. 
INDEX

Mots-clés : nature, culture, intention, théorie, méthode, interprétation

Keywords : nature, culture, intention, theory, method, interpretation

\section{AUTEUR}

MARC DOMINICY

Université libre de Bruxelles, LaDisco-Philixte, B-1050, Belgique 